\title{
A central approach to bound the number of crossings in a generalized configuration.
}

\author{
Bernardo M. Ábrego \\ Silvia Fernández-Merchant \\ Department of Mathematics, California State University at Northridge \\ Jesús Leaños \\ Gelasio Salazar \\ Instituto de Física, Universidad Autónoma de San Luis Potosí, México
}

\begin{abstract}
A generalized configuration is a set of $n$ points and $\left(\begin{array}{l}n \\ 2\end{array}\right)$ pseudolines such that each pseudoline passes through exactly two points, two pseudolines intersect exactly once, and no three pseudolines are concurrent. Following the approach of allowable sequences we prove a recursive inequality for the number of $(\leq k)$-sets for generalized configurations. As a consequence we improve the previously best known lower bound on the pseudolinear and rectilinear crossing numbers from $0.37968\left(\begin{array}{l}n \\ 4\end{array}\right)+\Theta\left(n^{3}\right)$ to $0.379972\left(\begin{array}{l}n \\ 4\end{array}\right)+\Theta\left(n^{3}\right)$.
\end{abstract}

Keywords: $k$-sets, $\leq k$-sets, rectilinear crossing number, pseudolinear crossing number, complete graphs

\section{Introduction}

A pseudoline is a curve in the projective plane whose removal does not disconnect it, or alternatively, a simple curve in the plane that extends infinitely 
on both directions. A generalized configuration consists of $\left(\begin{array}{l}n \\ 2\end{array}\right)$ pseudolines and $n$ points such that each pseudoline passes through exactly two points, two pseudolines intersect exactly once, and no three pseudolines are concurrent. A simple allowable sequence on $n$ points is a doubly infinite sequence $\Pi=\left(\ldots, \pi_{-1}, \pi_{0}, \pi_{1}, \ldots\right)$ of permutations on $n$ elements, such that any two consecutive permutations differ by a transposition of neighboring elements, and such that for every $j, \pi_{j}$ is the reversed permutation of $\pi_{j+\left(\begin{array}{l}n \\ 2\end{array}\right)}$. Then $\Pi$ is determined by $\pi_{0}, \ldots, \pi_{\left(\begin{array}{c}n \\ 2\end{array}\right)}$ and any given transposition occurs exactly once on this interval.

Allowable sequences were introduced by Goodman and Pollack [8], who proved a correspondence between the set of simple allowable sequences and the set of generalized configuration of points. When all the pseudolines are straight lines, the generalized configuration is completely determined by the set of points. In [2] two well-known concepts for configurations of points in general position in the plane were extended to generalized configurations: $k$ sets and rectilinear crossing number of $K_{n}$ (see [6] for more references and related problems). Given an $n$-point set $P$ and $k \leq n / 2$, a $k$-set is a subset of $k$ points in $P$ that can be separated from the rest by a straight line. The rectilinear crossing number of $K_{n}$, denoted $\overline{\mathrm{cr}}(n)$, is the maximum number of crossings determined by a complete geometric graph on $n$ vertices. In the more general setting, the $k$-sets of $\Pi$ are the subsets of $\{1,2, \ldots, n\}$ of size $k$ that occupy the first or last $k$ positions in a permutation of $\Pi$. These are determined by $k$-critical transpositions, that is, those that occur between elements in positions $k$ and $k+1$ or $n-k$ and $n-k+1$. Denote by $\mathcal{N}_{k}(\Pi)$ the number of $k$-critical transpositions of $\Pi$. Also let $\mathcal{N}_{\leq k}(\Pi)=\sum_{j=1}^{k} \mathcal{N}_{j}(\Pi)$ and $\mathcal{N}_{>k}(\Pi)=\left(\begin{array}{l}n \\ 2\end{array}\right)-\mathcal{N}_{\leq k}(\Pi)$, called the number of $(\leq k)$-sets and $(>k)$-sets of $\Pi$, respectively. Let $\widetilde{\mathrm{cr}}(\Pi)$ denote the number of pseudoline crossings in $\Pi$. Then the pseudolinear crossing number of $K_{n}$ is defined as $\widetilde{\mathrm{cr}}(n)=\min _{|\Pi|=n} \widetilde{\mathrm{cr}}(\Pi)$. Clearly $\widetilde{\mathrm{cr}}(n) \leq \overline{\mathrm{cr}}(n)$. The next relationship between $k$-sets and crossing numbers was proved in [2] and [9],

$$
\widetilde{\operatorname{cr}}(\Pi)=\sum_{k=1}^{\lfloor n / 2\rfloor}(n-2 k-1) \mathcal{N}_{\leq k}(\Pi)+\Theta\left(n^{3}\right) .
$$

The problem of determining the rectilinear crossing number was proposed by Erdös and Guy [7] and is equivalent to finding the minimum number of convex quadrilaterals in a set of $n$ points in general position. The best known upper bound, $\widetilde{\operatorname{cr}}(n) \leq \overline{\operatorname{cr}}(n) \leq 0.380548\left(\begin{array}{l}n \\ 4\end{array}\right)+\Theta\left(n^{3}\right)$, is attained by a recursive 
construction in [3] using as a starting point a suitable 90-point set. On the other hand, $\mathcal{N}_{\leq k}(\Pi)$ was bounded in [1] (and in [5] in the rectilinear case) by

$$
\mathcal{N}_{\leq k}(\Pi) \geq 3\left(\begin{array}{c}
k+1 \\
2
\end{array}\right)+3\left(\begin{array}{c}
k+1-\lfloor n / 3\rfloor \\
2
\end{array}\right)-\max \{0,(k-\lfloor n / 3\rfloor)(n-3\lfloor n / 3\rfloor)\} .
$$

This in turn implies the previously best known lower bound [1]: $\overline{\mathrm{cr}}(n) \geq$ $\widetilde{\operatorname{cr}}(n) \geq 0.37968\left(\begin{array}{l}n \\ 4\end{array}\right)+\Theta\left(n^{3}\right)$.

In this work we first bound the $(>k)$-sets in terms of the $k$-sets in Theorem 2.1. Using this theorem with (1) and (2), we obtain $\overline{\operatorname{cr}}(n) \geq \widetilde{\operatorname{cr}}(n) \geq \frac{277}{729}\left(\begin{array}{l}n \\ 4\end{array}\right)+$ $\Theta\left(n^{3}\right)>0.379972\left(\begin{array}{l}n \\ 4\end{array}\right)+\Theta\left(n^{3}\right)$. It is important to note that this is the first time that a lower bound on $\overline{\mathrm{cr}}(n)$ and $\widetilde{\mathrm{cr}}(n)$ follows from the central behavior of a generalized configuration (a bound on the $(>k$ )-sets, i.e., all $k$-sets with $k$ close to $n / 2$ ) rather than on its boundary behavior (a lower bound on the $(\leq k)$-sets, i.e. all $k$-sets with $k$ far from $n / 2)$.

\section{The Central Theorem}

Given a permutation $\pi_{j}$ of $\Pi$ and $k \leq n / 2$, we denote by $C\left(k, \pi_{j}\right)$, the set of elements in the middle $n-2 k$ positions of the permutation $\pi_{j}$. Let

$$
s_{k}=\min \left\{\left|C\left(k, \pi_{0}\right) \cap C\left(k, \pi_{i}\right)\right|: 0 \leq i \leq\left(\begin{array}{l}
n \\
2
\end{array}\right)\right\} .
$$

Theorem 2.1 Let $\Pi$ be a generalized configuration of $n$ points. Then

$$
\mathcal{N}_{>k}(\Pi) \leq(n-2 k-1) \mathcal{N}_{k}(\Pi)-\frac{s_{k}}{2}\left(\mathcal{N}_{k}(\Pi)-(n-1)\right)
$$

In [4] we prove Theorem 2.1 for $k=\lfloor n / 2\rfloor-1$. In fact, for such $k$ we prove $\mathcal{N}_{>k}(\Pi) \leq(n-2 k-1) \mathcal{N}_{k}(\Pi)$ which does not depend on $s_{k}$ but is not true for all $k$. Intuitively, to bound the number of transpositions in the middle $n-2 k-1$ positions of $\Pi$ it is important to know how many elements remain in the middle positions at any given time. This is why we must involve $s_{k}$. Given $\Pi$, we first modify it in such a way that the transpositions within the first or last $k$ positions remain intact. This transformation also guarantees that $\mathcal{N}_{>k}(\Pi), \mathcal{N}_{k}(\Pi)$, and $s_{k}$ do not change, and allows us to control the $(>k)$-critical transpositions to count them depending on $s_{k}$.

Because of the space limitations we cannot include the proof of the theorem here. It will appear in the full version of this paper. We choose instead to show the consequences of this result to the number of $(\leq k)$-sets and to the pseudolinear and rectilinear crossing numbers. 


\section{New Lower Bound for $\mathcal{N}_{\leq k}(\Pi)$}

Let $m=\lceil(4 n-2) / 9\rceil$. Define for every $n$ the following recursive sequence:

$$
\begin{aligned}
u_{m-1} & =3\left(\begin{array}{c}
m \\
2
\end{array}\right)+3\left(\begin{array}{c}
m-\lfloor n / 3\rfloor \\
2
\end{array}\right)-3\left(m-1-\left\lfloor\frac{n}{3}\right\rfloor\right)\left(\frac{n}{3}-\left\lfloor\frac{n}{3}\right\rfloor\right) \text { and } \\
u_{k} & =\left\lceil\frac{1}{n-2 k}\left(\left(\begin{array}{l}
n \\
2
\end{array}\right)+(n-2 k-1) u_{k-1}\right)\right\rceil \text { for } k \geq m .
\end{aligned}
$$

The next lemmas give useful estimates for the sequence $u_{k}$ and are needed for the proofs of Theorem 3.3 and Corollary 3.4. They can be proved by elementary inductive arguments.

Lemma 3.1 For any $k, m-1 \leq k \leq(n-3) / 2$ :

$$
3 \sqrt{1-\frac{2 k+5 / 2}{n}} \leq \frac{\left(\begin{array}{l}
n \\
2
\end{array}\right)-u_{k}}{\left(\begin{array}{l}
n \\
2
\end{array}\right)-u_{m-1}} \leq 3 \sqrt{1-\frac{2 k}{n}} .
$$

Lemma 3.2 For any $k, m \leq k \leq(n-3) / 2$ :

$$
3 \sqrt{1-\frac{2 k+5 / 2}{n}}\left(\left(\begin{array}{l}
n \\
2
\end{array}\right)-u_{m-1}\right) \geq(n-1)(n-2 k-1) .
$$

This is the new lower bound that follows once Theorem 2.1 is established:

Theorem 3.3 For any generalized configuration $\Pi$ and any $k, m-1 \leq k \leq$ $(n-2) / 2$ we have $\mathcal{N}_{\leq k}(\Pi) \geq u_{k}$.

Proof of Theorem 3.3. We proceed by induction on $k$. If $k=m-1$ the result is true by the bound in (2). Assume $k \geq m$ and $\mathcal{N}_{\leq k-1}(\Pi) \geq u_{k-1}$. From Theorem 2.1, if $s=0$ or $\mathcal{N}_{k}(\Pi) \geq n-1$ then $\mathcal{N}_{>k}(\Pi) \leq(n-2 k-1) \mathcal{N}_{k}(\Pi)$. Thus

$$
\left(\begin{array}{l}
n \\
2
\end{array}\right)-\mathcal{N}_{\leq k}(\Pi) \leq(n-2 k-1)\left(\mathcal{N}_{\leq k}(\Pi)-\mathcal{N}_{\leq k-1}(\Pi)\right),
$$

and then by induction

$$
\mathcal{N}_{\leq k}(\Pi) \geq \frac{1}{n-2 k}\left(\left(\begin{array}{l}
n \\
2
\end{array}\right)+(n-2 k-1) u_{k-1}\right) .
$$

This implies that $\mathcal{N}_{\leq k}(\Pi) \geq u_{k}$ by definition of the sequence $u_{k}$. 
Now assume $s_{k}>0$ and $\mathcal{N}_{k}(\Pi)<n-1$. It is easy to see that (c.f. [9]) $\mathcal{N}_{k}(\Pi) \geq 2 k+1$, thus $k \leq(n-3) / 2$. From Theorem 2.1 we have that

$$
\mathcal{N}_{>k}(\Pi) \leq\left(n-2 k-1-\frac{s_{k}}{2}\right) \mathcal{N}_{k}(\Pi)+\frac{s_{k}}{2}(n-1)
$$

and since $n-2 k-1-s_{k} / 2>n-2 k-1-s_{k} \geq 0$ and $\mathcal{N}_{k}(\Pi)<n-1$, then

$$
\mathcal{N}_{>k}(\Pi)<\left(n-2 k-1-\frac{s_{k}}{2}\right)(n-1)+\frac{s_{k}}{2}(n-1)=(n-1)(n-2 k-1) .
$$

Therefore

$$
\mathcal{N}_{\leq k}(\Pi)>\left(\begin{array}{l}
n \\
2
\end{array}\right)-(n-1)(n-2 k-1) .
$$

Then by Lemmas 3.1 and 3.2 we have that $\mathcal{N}_{\leq k}(\Pi) \geq u_{k}$.

Corollary 3.4 For any generalized configuration $\Pi$ and any $k, m-1 \leq k \leq$ $(n-2) / 2$ we have

$$
\mathcal{N}_{\leq k}(\Pi) \geq\left(\begin{array}{l}
n \\
2
\end{array}\right)-\frac{1}{9} \sqrt{1-\frac{2 k}{n}}\left(5 n^{2}-25 n+4\right)
$$

Proof. Follows directly from Theorem 3.3, Lemma 3.1, and the fact that $u_{m-1} \leq 3\left(\begin{array}{c}m \\ 2\end{array}\right)+3\left(\begin{array}{c}m-\lfloor n / 3\rfloor \\ 2\end{array}\right) \leq 3\left(\begin{array}{c}(4 n+6) / 9 \\ 2\end{array}\right)+3\left(\begin{array}{c}(n+10) / 9 \\ 2\end{array}\right)$.

Corollary $3.5 \overline{\operatorname{cr}}(n) \geq \widetilde{\operatorname{cr}}(n) \geq \frac{277}{729}\left(\begin{array}{l}n \\ 4\end{array}\right)+\Theta\left(n^{3}\right)>0.379972\left(\begin{array}{l}n \\ 4\end{array}\right)+\Theta\left(n^{3}\right)$.

Proof. According to (1), if $\Pi$ is a generalized configuration on $n$ points then

$$
\widetilde{c r}(\Pi)=\left(\begin{array}{l}
n \\
4
\end{array}\right)\left(24 \sum_{k=1}^{\lfloor n / 2\rfloor-1} \frac{1}{n}\left(1-\frac{2 k}{n}\right) \frac{\mathcal{N}_{\leq k}(\Pi)}{n^{2}}\right)+\Theta\left(n^{3}\right)
$$

Now, using (2) we know that for $1 \leq k \leq m-1$,

$$
\frac{\mathcal{N}_{\leq k}(\Pi)}{n^{2}} \geq \frac{3}{2}\left(\frac{k}{n}\right)^{2}+\frac{3}{2} \max \left(0, \frac{k}{n}-\frac{1}{3}\right)^{2}-\Theta\left(\frac{1}{n}\right) .
$$

Similarly, if $m \leq k \leq(n-2) / 2$ then by Corollary 3.4 ,

$$
\frac{\mathcal{N}_{\leq k}(\Pi)}{n^{2}} \geq \frac{1}{2}-\frac{5}{9} \sqrt{1-\frac{2 k}{n}}+\Theta\left(\frac{1}{n}\right) .
$$


Therefore

$$
\begin{aligned}
\widetilde{c r}(\Pi) & \geq\left(\begin{array}{l}
n \\
4
\end{array}\right)\left(24 \int_{0}^{4 / 9} \frac{3}{2}(1-2 x)\left(x^{2}+\max \left(0, x-\frac{1}{3}\right)^{2}\right) d x\right) \\
& +\left(\begin{array}{l}
n \\
4
\end{array}\right)\left(24 \int_{4 / 9}^{1 / 2}(1-2 x)\left(\frac{1}{2}-\frac{5}{9} \sqrt{1-2 x}\right) d x\right)+\Theta\left(n^{3}\right) \\
& \geq\left(\begin{array}{l}
n \\
4
\end{array}\right)\left(\frac{86}{243}+\frac{19}{729}\right)+\Theta\left(n^{3}\right)=\frac{277}{729}\left(\begin{array}{l}
n \\
4
\end{array}\right)+\Theta\left(n^{3}\right) .
\end{aligned}
$$

\section{References}

[1] B. M. Ábrego, J. Balogh, S. Fernández-Merchant, J. Leaños, and G. Salazar. An extended lower bound on the number of $(\leq k)$-edges to generalized configurations of points and the pseudolinear crossing number of $K_{n}$. Submitted (2006).

[2] B. M. Ábrego and S. Fernández-Merchant, A lower bound for the rectilinear crossing number, Graphs and Comb. 21 (2005), 293-300.

[3] B. M. Ábrego and S. Fernández-Merchant. Geometric drawings of $K_{n}$ with few crossings. J. Combin. Theory Ser. A 114 (2007), 373-379.

[4] B. M. Ábrego, S. Fernández-Merchant, J. Leaños, and G. Salazar. The maximum number of halving lines and the rectilinear crossing number of $K_{n}$ for $n \leq 27$. Preprint (2007).

[5] O. Aichholzer, J. García, D. Orden, and P. Ramos, New lower bounds for the number of $(\leq k)$-edges and the rectilinear crossing number of $K_{n}$. Discrete and Comp. Geom., to appear.

[6] P. Brass, W. Moser and J. Pach, Research Problems in Discrete Geometry, Springer-Verlag, New York, 2005.

[7] P. Erdős and R. K. Guy, Crossing number problems, Amer. Math. Monthly 80 (1973), 52-58.

[8] J. E. Goodman, R. Pollack, On the combinatorial classification of nondegenerate configurations in the plane, J. Combin. Theory Ser. A 29 (1980), 220-235.

[9] L. Lovász, K. Vesztergombi, U. Wagner, E. Welzl, Convex quadrilaterals and $k$-sets. In: Pach, J. editor: Towards a theory of geometric graphs, Contemporary Mathematics Series, 342, AMS 2004, 139-148. 